\title{
Differences in the distribution of F-actin in outer hair cells along the organ of Corti
}

\author{
Peter R. Thorne, Lynn Carlisle, Gary Zajic. Jochen Schacht and Richard A. Altschuler \\ Kresge Hearing Research Institute, University of Michigan Medical Center. Ann Arbor, MI 48109. U.S.A.
}

(Received 16 March 1987; accepted 27 June 1987)

\begin{abstract}
There is evidence of differences in the structure, innervation and physiological responses between outer hair cells (OHCs) of the basal and apical turns of the mammalian cochlea. In this study we have used rhodamine-labelled phalloidin to investigate the differential distribution of F-actin in OHCs along the organ of Corti of the guinea pig. Isolated OHCs and surface preparations and cryosections of the organ of Corti were studied. F-actin was observed in stereocilia and the cuticular plate of all $O H C s$. In addition. some OHCs had a network of F-actin extending from the cuticular plate towards the nucleus. This infracuticular network was observed in most OHCs of the apical cochlear turns but was not seen in any OHCs of the basal turn. These microstructural differences between OHCs of the base and apex could be related to differences in OHC function between the apical and basal portions of the cochlea.
\end{abstract}

Rhodamine-labelled phalloidin; F-actin: Organ of Corti: Hair cell. outer

\section{Introduction}

There is an increasing body of literature demonstrating that the outer hair cells (OHCs) of the mammalian cochlea do not comprise a homogeneous cell population, but that substantial differences exist in the structure and physiological responses of these cells from the base to apex of the organ of Corti. Structural differences that have been noted in OHCs along the organ of Corti include the length of the cell body (Smith, 1968), and the number, length (Strelioff and Flock, 1984; Wright, 1984) and stiffness of stereocilia (Strelioff and Flock, 1984). OHCs in the base also have a substantial efferent innervation and this diminishes apically where OHICs receive more afferent nerve fibres (Spoendlin, 1979). There are also temporal differences in the development of the

Correspondence to: P.R. Thorne (present address), Department of Pathology, University of Auckland School of Medicine. Private Bag. Auckland. New Zealand. innervation of OHCs along the organ of Corti (Pujol and Lenoir, 1986). Recent studies have shown different physiological responses of OHCs at different locations in the cochlea. OHCs in the apical turns show both $\mathrm{AC}$ and $\mathrm{DC}$ receptor potentials in response to acoustic stimulation at their best frequency (Dallos, 1985a). OHCs in the base of the cochlea have $\mathrm{AC}$ receptor potentials but do not exhibit DC receptor potentials to high frequency stimulation, except at very high sound levels (Russell and Cody. 1985). This indicates that OHCs in the apical turns but not those in the basal turns are depolarised during sound stimulation (Dallos, 1985a). Thus, OHCs in the base may not be able to initiate transmitter release at their synaptic region.

The function of OHCs is not yet understood. Presumably, they are able to provide some direct sensory input to the central nervous system through their sparse afferent innervation. It has also been suggested that they may alter the sensitivity and frequency selectivity of the mechanical response of the cochlear partition by an active process (Davis, 1983; Neely and Kim, 1983). Re- 
cently, OHCs have been shown to change their length in vitro following electrical stimulation (Brownell et al., 1986; Kachar et al., 1986) or depolarizing concentrations of potassium in the incubation medium (Zenner et al., 1985) or the application of calcium to permeabilized cells in the presence of ATP (Flock et al., 1986; Schacht and Zenner, 1986; Zenner, 1986b). Contractile proteins have been identified in $\mathrm{OHCs}$ which could provide these cells with a mechanism for motility (Flock, 1985; Flock et al., 1986).

The differences in the physiological responses of OHCs along the organ of Corti led Dallos (1985b) to speculate that apical OHCs act as receptor cells whereas those in the basal turn with fewer afferent fibres, have a more predominant motor or effector function. It is reasonable to assume that such differences in function would be associated with differences in the organization of the structures involved in this effector or motile function. Contraction in OHCs may involve an actin-myosin system (Flock et al., 1986; Zenner, $1986 \mathrm{a}, \mathrm{b})$ and the localization and organization of various contractile proteins in hair cells have been well documented (see reviews by Drenkhahn et al.. 1985; Flock, 1985; Saunders et al., 1985; Tilney et al., 1985; Zenner, 1986a). There is, however, no information on any quantitative differences in the distribution of cytoskeletal proteins in cells along the organ of Corti or among the rows of hair cells.

The distribution of actin filaments (F-actin) in hair cells along the cochlea and in isolated $\mathrm{OHCs}$ was chosen for study because it has been found in hair cells of the mammalian cochlea, and is considered to be a major protein of motile elements. It is localized in the stereocilia, cuticular plate and the synaptic region of hair cells (Flock and Cheung, 1977; Tilney et al., 1980; Slepecky and Chamberlain, 1985; Flock et al.. 1986). In addition, actin filaments have been demonstrated extending from the cuticular plate to the perinuclear region (Zenner, 1980, 1986a,b; Zenner and Drenkhahn, 1986) and also along the lateral margins of OHCs (Flock et al., 1986). In the present study actin was visualized with a specific marker for F-actin, rhodamine-labelled phalloidin (Faulstich et al., 1983), and the results indicate that there are differences in the localization of F-actin in OHCs along the organ of Corti.

\section{Materials and Methods}

Hair cells of pigmented guinea pigs $(150-300 \mathrm{~g})$ were studied by four different procedures: light microscopy of isolated OHCs, surface preparations of the organ of Corti, cryosections of the cochlea and transmission electron microscopy of the organ of Corti.

\section{Isolated hair cells}

Isolated hair cells were studied either fixed or non-fixed ('living'). The isolation procedure was essentially the same as that described by Zenner et al. (1985) and Zajic and Schacht (1987). The animal was decapitated and the apical or other selected turn of the cochlea was removed immediately and transferred to a $60 \times 15 \mathrm{~mm}$ Petri dish containing a drop of tissue culture medium (Hanks' balanced salt solution (HBSS); Gibco Laboratories, U.S.A.; osmolarity adjusted to 300 mOsm with $\mathrm{NaCl}$ ) with $1 \mathrm{mg}$ collagenase/ml (Type 14, Sigma Chemical Company, St Louis, MO, U.S.A.) at room temperature. The organ of Corti was incubated in this medium for $10 \mathrm{~min}$ and subsequently transferred into fresh HBSS. Hair cells were then teased from the basilar membrane using fine tungsten needles and transferred to a glass well-slide using a $0.1 \mathrm{ml}$ glass syringe. Cells were incubated with $33 \mathrm{nM}$ rhodamine-labelled phalloidin (Molecular Probes Inc, Eugene, OR, U.S.A.) in HBSS for $20 \mathrm{~min}$ in the dark at room temperature, then washed twice in HBSS, covered with a coverslip and studied under the light microscope (see below). Some hair cells were pretreated with saponin $(12 \mu \mathrm{g} / \mathrm{ml}$ HBSS; Sigma Chemical Co., St. Louis, MO, U.S.A.) for $10 \mathrm{~min}$ in calcium-free media and others were transferred into a drop of fixative $(4 \%$ paraformaldehyde in $0.1 \mathrm{M}$ phosphate buffer, $\mathrm{pH}$ 7.4) for $20 \mathrm{~min}$. These cells were then washed in buffer, and incubated with $33 \mathrm{nM}$ phalloidin in HBSS for $20 \mathrm{~min}$ and again washed twice in buffer. After covering the well-slide with a coverslip the cells were studied by light microscopy.

\section{Surface preparations}

Animals were anaesthetized with sodium pentobarbitone $(50 \mathrm{mg} / \mathrm{kg}$ ) and then decapitated. Both bullae werc removed rapidly and opened, then immersed in fixative (4\% paraformaldehyde 
and $0.1 \%$ glutaraldehyde in $0.1 \mathrm{M}$ phosphate buffer at $\mathrm{pH}$ 7.4) and the bony cochlea was partially removed to expose the membranous portions. Each cochlea was also perfused with fixative through the round and oval windows. The cochleae were left in fixative for $1 \mathrm{~h}$, washed in buffer $(0.1 \mathrm{M}$ phosphate, $\mathrm{pH}$ 7.4) and then transferred to phosphate buffered saline (PBS, pH 7.4) for partial dissection. Under the dissection microscope the bony wall was removed completely to expose the spiralling organ of Corti and modiolus which was then incubated in $33 \mathrm{nM}$ phalloidin in PBS in the dark with gentle rotation for $24 \mathrm{~h}$ at room temperature. The coils were washed twice in PBS and dissected to remove the organ of Corti in half-turn segments. These were mounted on glass slides, with the endolymphatic surface facing upward, covered with a coverslip and viewed by light microscopy.

As a control for non-specific staining by rhodamine the organs of Corti from two animals were prepared as surface preparations and treated with rhodamine-labelled $\alpha$-bungarotoxin (Molecular Probes Inc., Eugene, OR, U.S.A.).

\section{Crrosections}

Cochleae were fixed according to the schedule above. Following the fixation procedure they were dissected to remove the bony cochlear wall but the organ of Corti was left attached to the modiolar bone. The coils were decalcified for $16-20 \mathrm{~h}$ in a solution of $2 \%$ EDTA in PBS with $6 \%$ sucrose added as a cryoprotectant. Coils were then frozen in Tissue-Tek (Miles Scientific, Naperville, IL, U.S.A.) on a cryostat chuck and sections $10 \mu \mathrm{m}$ in thickness were cut on a cryostat (Hacker Instruments Inc., Fairfield, NJ, U.S.A.). These sections were mounted on glass slides and then incubated with $33 \mathrm{nM}$ phalloidin in PBS for $24 \mathrm{~h}$ at room temperature. Sections were then washed and viewed by light microscopy as described below.

\section{Electron microscopy}

After opening the bullae, cochleae were perfused immediately through the round and oval windows with $2.5 \%$ glutaraldehyde in $0.1 \mathrm{M}$ phosphate buffer ( $\mathrm{pH} 7.4$ ) and left immersed in fixative for $4 \mathrm{~h}$. After washing in $0.1 \mathrm{M}$ phosphate buffer they were post-fixed in $1 \% \mathrm{OsO}_{4}$ in $0.1 \mathrm{M}$ phosphate buffer ( $\mathrm{pH}$ 6.4) on ice for $30 \mathrm{~min}$. washed in buffer and then stained with $0.5 \%$ uranyl acetate in $50 \%$ ethanol for $15 \mathrm{~min}$. Each cochlea was dissected in $50 \%$ ethanol and pieces of the organ of Corti removed, dehydrated in ascending concentrations of ethanol, infiltrated with resin and embedded in flat molds. Gold-silver thin sections were cut on a Reichert Ultracut microtome using a diamond knife and then studied using a Jeol 1200 EX electron microscope.

\section{Light microscopy}

Isolated hair cells, surface preparations and cryostat sections were studied by light microscopy (Leitz Orthomat) using either bright field or epifluorescence optics (460-470 nm excitation filter and 510-550 nm objective filter) with $25-100 \times$ oil immersion objectives. Photographs were taken at magnifications of $250-1000 \times$ using Ektachrome color slide film (160 ASA) or Kodak black and white Tri-X Pan film (400 ASA).

\section{Results}

\section{Isolated hair cells}

Hair cells in vitro had a cylindrical shape with a clear cytoplasm, normally positioned organelles and a basally located nucleus. Such hair cells were considered morphologically normal according to the criteria established by Zajic and Schacht (1987). Hair cells that did not satisfy these criteria were excluded from this study.

Phalloidin labelling was observed in the stereocilia, cuticular plate and occasionally in the extreme basal pole of the cell (Fig. 1). In addition, in some OHCs a structure was stained that extended from beneath the cuticular plate towards the perinuclear regions for variable depths (Fig. 1). This structure usually had the appearance of a dense core that spiralled in the infracuticular region but straightened towards the supranuclear region of the hair cell. The infracuticular network of actin was not apparent in all OHCs but was observed in OHCs that had been permeabilized with saponin prior to the incubation in phalloidin. Hair cells also showed some fluorescence along the lateral margins of the cell.

The appearance of the fixed isolated hair cells was similar although the cells were generally more 


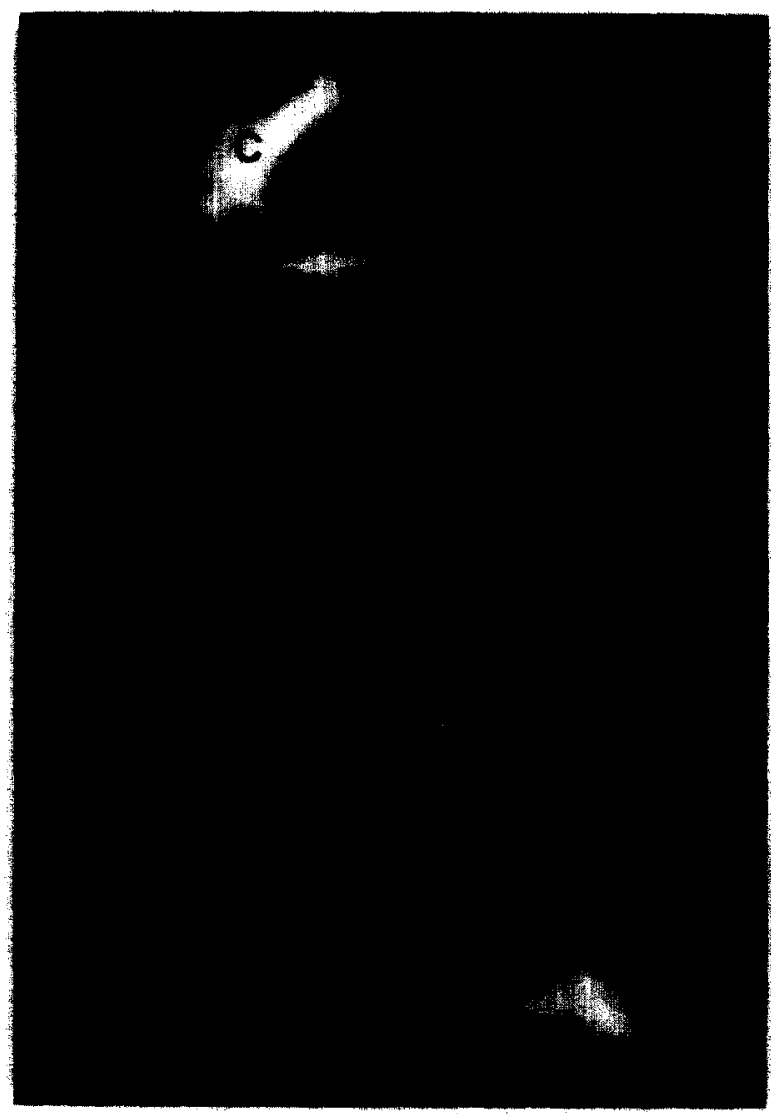

Fig. 1. A non-fixed isolated $\mathrm{OHC}$ (not saponin treated) shown by fluorescence microscopy. Phalloidin labelling is seen in the sterencilia (S). cuticular plate ( $)$, as a spital structure (large arrow) extending into the cytoplasm from beneath the cuticular plate, and the synaptic region (SY), and along the lateral margins of the cell (small arrows). Bar, $10 \mu \mathrm{m}$.

granular and showed a denser cytoplasm. Staining was observed in the cuticular plate region, stereocilia, basal end and in the cytoplasm, essentially as seen in the non-fixed OHC. While the cytoplasmic network of phalloidin staining was not seen in every fixed cell it was more consistently observed than in non-fixed, normal appearing OHCs.

\section{Surface preparations}

Staining of the organ of Corti with phalloidin revealed two clear patterns of distribution of $F$ actin in hair cells. One staining pattern was seen in all $\mathrm{OHCs}$ and was confined to stereocilia, cuticular plate, a faint staining around the entire circumference, and at the base of the cell. A second pattern, seen only in some OHCs, consisted of a core of labelled $F$-actin extending from the cuticular plate towards the perinuclear region of the cell in addition to the other areas of staining. These observations essentially confirmed the findings in isolated OHCs. Inner hair cells (IHCs) showed staining in the stereocilia and cuticular plate but nowhere else.

The cuticular plate of both OHCs and IHCs showed a very distinctive pattern of phalloidin distribution when viewed from their endolymphatic surface. In a number of areas of the cuticular plate the staining did not reach the margins of the cell but instead the staining gave the appearance of a scallop shape (Fig. 2). Particularly noticeable was an absence of staining in the cuticular-free region on the lateral margin of the cells.

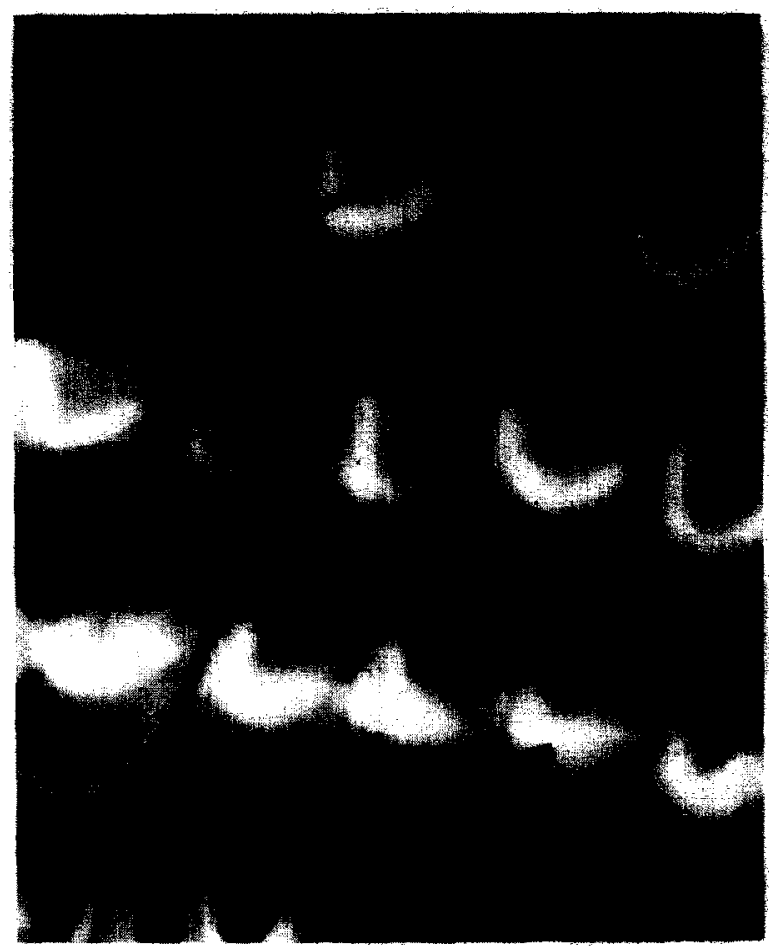

Fig. 2. The surface of the organ of Corti seen by fluorescence microscopy. Three rows of OHCs $(1,2,3)$ are shown focussed at the reticular lamina. Phalloidin staining is seen in the cuticular plate $(C)$ but is absent from the cuticular free region (large arrow) and at various locations around the margins (small arrows). Note the intense staining of radiating filaments in the Deiter's cells (open arrow) beyond the third row OHCs. Surface preparation. Bar. $10 \mathrm{~km}$. 

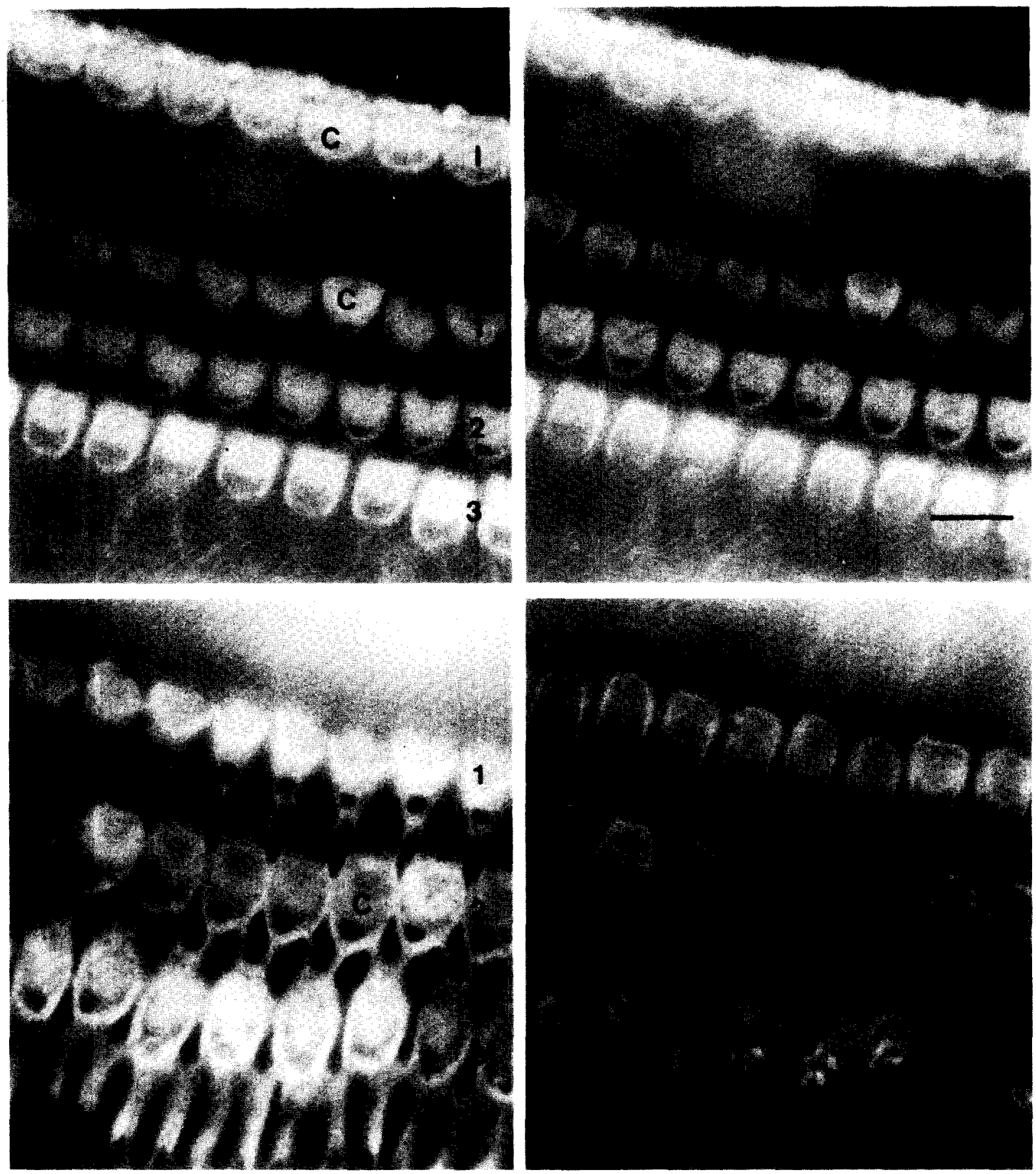

Fig. 3. The organ of Corti of the first (A) and the third (B) turns focussed at the reticular lamina (left) and beneath the cuticular plate (right). In both turns phalloidin staining is seen in cuticular plates (C) of IHCs (I) and the three rows of OHCs (1.2.3). In OHCs of the third turn additional staining is seen deeper in the lateral portion of the cell (arrows) but such structures are not observed in the first turn OHCs. Surface preparation. Bar, $10 \mu \mathrm{m}$. 
A striking observation in the surface preparations was the distribution of the second pattern of staining. The infracuticular network of F-actin was easily visible by focussing at different levels in the hair cell. It was present in OHCs but was never seen in IHCs at any location along the organ of Corti. Furthermore, the presence and structural details of the network were dependent upon the location of the OHCs in the organ of Corti. OHCs in the base of the cochlea did not have this network (Fig. 3) but progressively along the organ of Corti from the second turn to the apex it appeared first in OHCs of the third row in the second turn, then in the second row and finally in the first row OHCs. In the third turn all rows of OHCs showed this network in some form although in the first row it was very faint and still not visible in all cells (Fig. 3). In the extreme apex of the cochlea this pattern became less consistent
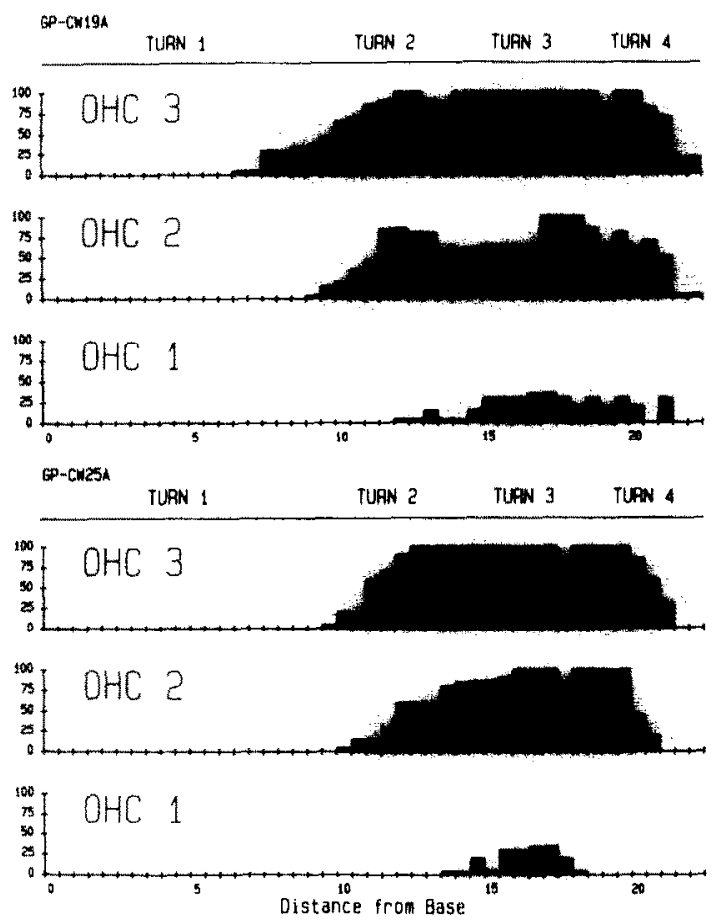

Fig. 4. The quantitative distribution of the infracuticular network or actin core in OHCs along the organ of Corti of two animals. In each cochlea the percentage of hair cells of the three rows of $\mathrm{OHCs}$ in the field, at $1000 \times$ magnification. showing any form of the network regardless of size, is represented as a function of distance ( $\mathrm{mm}$ ) from the basal end of the organ of Corti. and hair cells of all outer rows were occasionally lacking the network. Fig. 4 shows the distribution of OHCs along the organ of Corti with this infracuticular filamentous network, quantitatively assessed by determining the percentage of cells of each row of the organ of Corti that showed the network.

The form of the infracuticular structure also differed according to the position of the vell along the cochlea. In OHCs of the apex and in the third row of other turns it extended for a long distance down into the cell and appeared as a large structure almost circular in cross-section (Fig. 5). The network in OHCs of the second turn and in the first row in the apical turns was thinner and less spiralled. Those OHCs that showed a distinct, thick network of filaments extending into the cell also had a distinct circular ring of phalloidin staining slightly beneath the cuticular plate and a further region of staining connecting these two structures (Fig. 5). The impression was that the filaments extending into the cell formed a continuous structure with the ring in the cuticular plate.

Most of the supporting cells showed some degree of staining. The headplates of the inner and outer pillar cells were intensely stained as were the base or nuclear region of these cells. In addition, the phalangeal processes of the pillar cells and Deiter's cells showed heavy staining and the headplates of the Deiter's cells had an area of staining around their junctions with the hair cells. The Deiter's cells abutting the third row of hair cells had a distinctive pattern of filaments which seemed to be close to the endolymphatic surface of the cell and radiated, from their junction with the hair cell, laterally towards the Hensen cells (Figs. 2 and 5). Prominent staining was seen in the area of the border cells medial to the IHCs.

Organs of Corti incubated with $\alpha$-bungarotoxin did not show any fluorescent staining.

\section{Cryosections}

The presence of F-actin throughout the organ of Corti was confirmed by phalloidin staining of cryosections (Fig. 6). The arrangement of the infracuticular structure in OHCs of the various turns was clearly visible by this method. The network in OHCs of the third and second row in the apical 

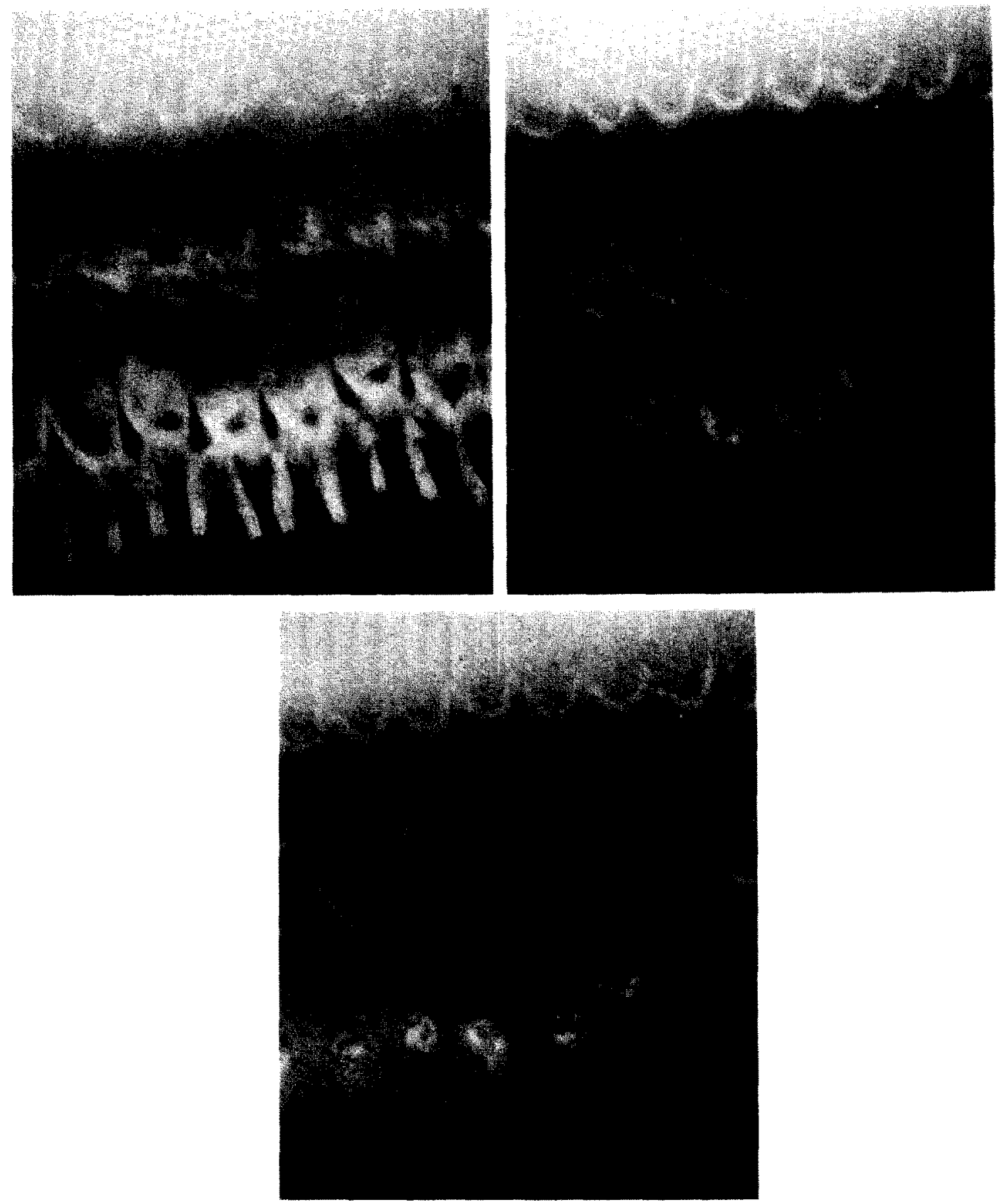

Fig. 5. The OHCs $(1,2,3)$ of the third turn of the organ of Corti stained with phalloidin and focussed at the reticular lamina (A) and at successively deeper layers ( $B$ and $C$ ). In addition to the staining at the cuticular plate, a brightly staining core is seen extending into the hair cell body (large arrow). This core has a circular cross-section in the third row OHCs (3) and is far more prominent in these cells than the other two rows. In the second and third rows a ring of staining can be seen at a level beneath the cuticular plate (small arrows). This ring seems to be associated with the core extending into the cell. Surface preparation. Bar. $10 \mu m$. 

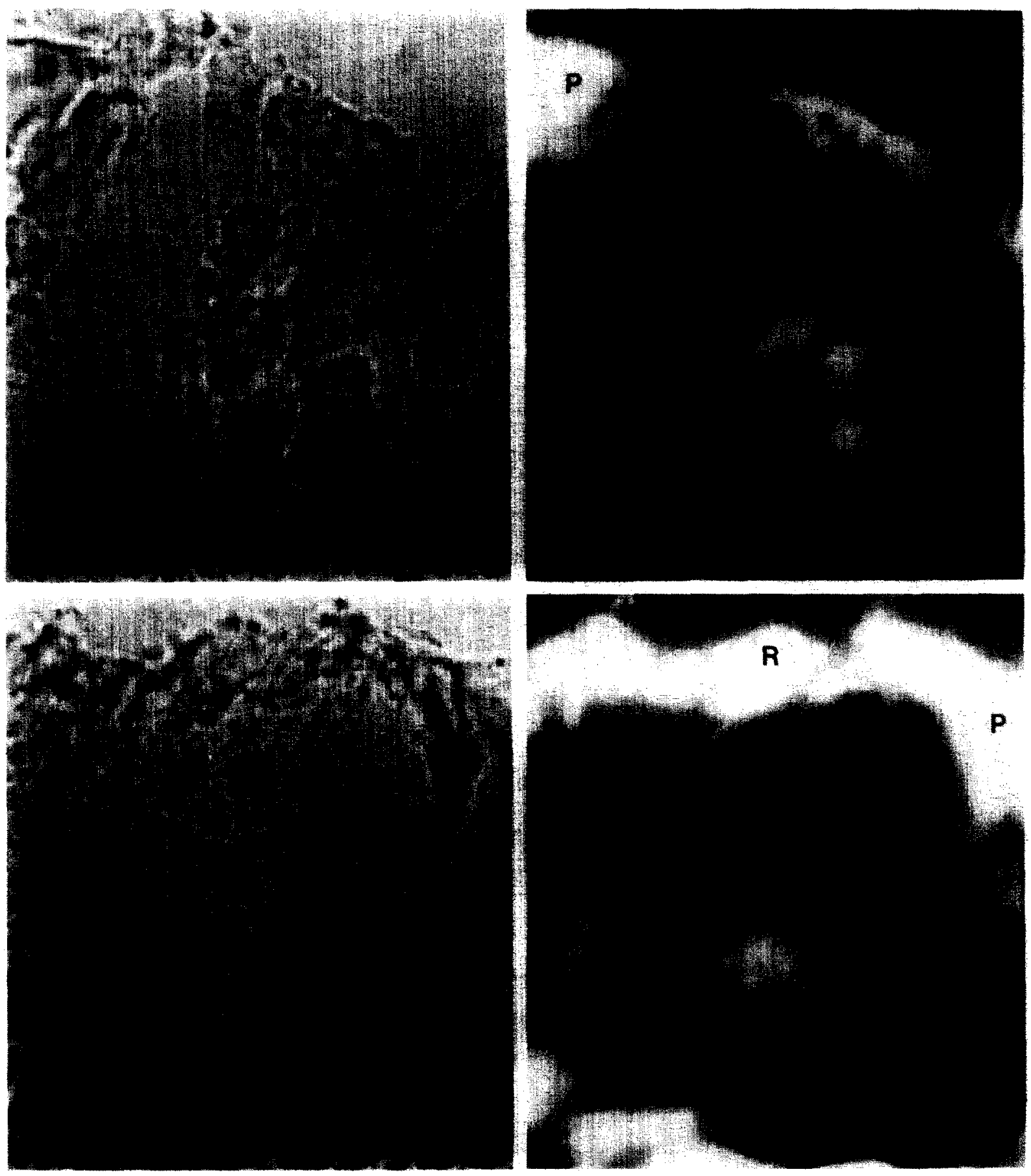

Fig. 6. Cryosections of the first (A) and third (B) turns seen in bright field (left) and by fuorescence microscopy (right). In the first turn phalloidin staining is seen in the reticular lamina $(R)$ and synaptic region (S) of the three rows of OHCs (1, 2, 3). Staining is also apparent in the pillar (P) cells. In the third turn a distinet core of staining (arrow) is seen spiralling from the cuticular plate towards the nucleus of an OHC of the third row. No such staining is seen in OHCs of the first turn. Cryosections. Bar, $10 \mu \mathrm{m}$. 
turns was thick, had a pronounced spiral and extended deep into the cell, whereas those of the first row in the same turns were thinner and did not extend as far into the cell. OHCs in hasal locations showed no evidence of the network. Intense staining in the reticular lamina region of the organ of Corti obscured any detail of the organization of F-actin in the cuticular plate area of the hair cells. No staining was observed within the cell body of IHCs.

\section{Transmission electron microscopy}

Transmission electron microscopy revealed collections of filaments and structures composed of dense-staining material resembling the cuticular plate at various locations in the cytoplasm (Fig. 7) of OHCs in all cochlear turns except the basal turn. In these cells, bundles of filaments were observed predominantly in the infra-cuticular region and were often continuous with the cuticular plate. In some OHCs, bundles of filaments were observed throughout the central axis of the cell body and extending between the cuticular plate and the nucleus. Infracuticular filaments were most obvious in the 2 nd and 3 rd row OHCs in the 3 rd and 4th cochlear turns. They were observed in association with mitochondria, microtubules and smooth endoplasmic reticulum (Fig. 7).

\section{Discussion}

F-actin in hair cells has a differential distribution among $\mathrm{OHCs}$ along the organ of Corti. The presence and structural detail of an infracuticular network of F-actin depends upon both the radial and longitudinal position of $\mathrm{OHCs}$ in the organ of Corti. In contrast, there are no apparent differences in the distribution of F-actin in stereocilia, cuticular plate, and the subplasmalemmal and synaptic regions of OHCs along the organ of Corti.

The finding of F-actin in the infracuticular region of isolated $\mathrm{OHCs}$ confirms the results of previous studies using anti-actin antibodies
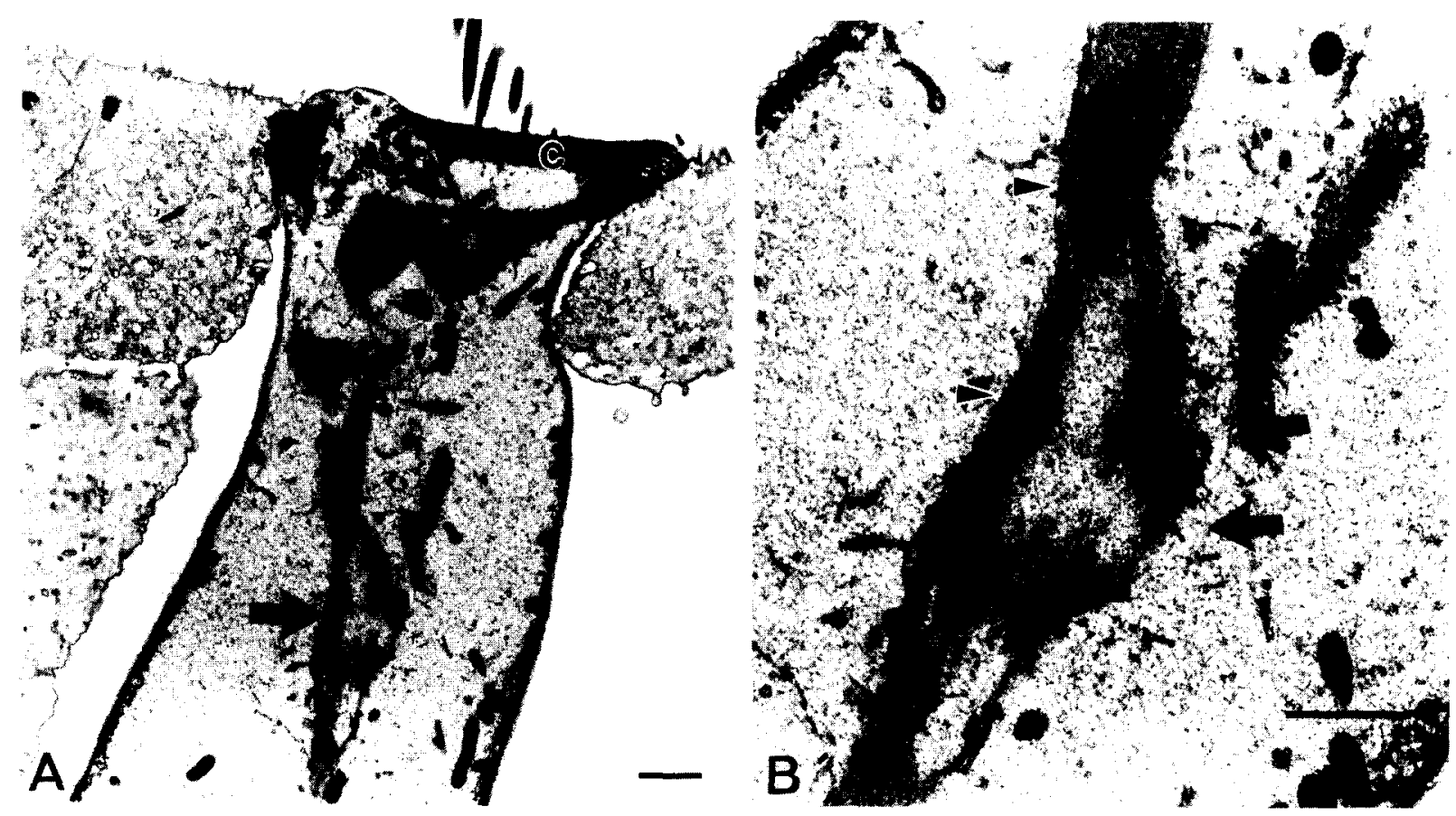

Fig. 7. (A) An $\mathrm{OHC}$ from the third cochlear turn showing the cuticular plate $(\mathrm{C})$ with areas extending into the infracuticular region (large arrow). Bar, $10 \mu \mathrm{m}$. (B) These areas shown at higher magnification have a filamentous and laminated appearance and are associated with mitochondria, cisternae (arrowheads) and microtubules (arrows). Transmission electron microscopy. Bar, $1 \mu \mathrm{m}$. 
(Zenner, 1986a) and phalloidin (Zenner, 1986b; Zenner and Drenkhahn. 1986). This study extends these observations to describe the differential distribution of the infracuticular network in OHCs along the organ of Corti. The infracuticular network corresponds presumably to the islands of actin' described in OHCs by Flock et al. (1982) using anti-actin antibodies and cryosections.

The electron microscopic evidence can be taken to support the differential distribution of F-actin. It revealed filaments of electron-dense material resembling the cuticular plate in the infracuticular region of OHCs from all cochlear turns but the basal turn. This structure would be equivalent to that stained by phalloidin. Previous authors have noted an extension of cuticular plate material into the cell cytoplasm, particularly in guinea pigs exposed to noise (Spoendlin, 1970; Lim and Melnick, 1971). As the results of the present study show, the appearance of the infracuticular network in any $\mathrm{OHC}$ depends upon its location along the organ of Corti. Thus, without a detailed comparison of the hair cells of noise-exposed animals with unexposed controls, it is not possible to determine whether the infracuticular network appears or increases in size in damaged cells. A well organized laminated filamentous structure extending from the cuticular plate to the base of the cell has been demonstrated in vestibular hair cells of the lamprey (Lowenstein and Osborne, 1964; Hoshino, 1975).

In the present study it was found that the staining of infracuticular actin occurred less consistently in non-fixed ('living') $\mathrm{OHCs}$ with a normal morphology, than the other preparations. Phallotoxins do not readily cross membranes of living cells (Barak et al., 1981) which may explain this variable staining. This is supported by the far more consistent staining pattern seen in isolated $\mathrm{OHCs}$ that were either pre-treated with saponin or fixed to improve membrane permeability.

Although an effect of the various treatments (fixation, saponin and phalloidin) on the extent of polymerization of actin in the infracuticular region of $\mathrm{OHCs}$ might explain the variable staining and differences in its distribution along the organ of Corti, this would seem unlikely considering that filaments were observed in all preparations (albeit inconsistently in non-fixed isolated OHCs). Saponin treatment was very mild and did not result in any morphological change in OHCs as seen by light microscopy. There does not appear to be any evidence that fixation with aldehydes affects the polymerization of actin (Hayat, 1981) although the destruction of actin filaments by osmium tetroxide fixation after aldehydes is well documented (Maupin-Szamier and Pollard, 1978; Pollard et al., 1984). This latter effect can be reduced by using osmium tetroxide in phosphate buffer at low $\mathrm{pH}$ and temperature (Tilney et al., 1980 ) as was used for fixation for electron microscopy in the present study. Zenner (1986b) has reported an enhancement of rhodamine-phalloidin staining in the infracuticular regions when demembranated isolated OHCs are induced to contract by the application of AIP and calcium. It was concluded that contraction of $\mathrm{OHCs}$ is associatcd with a rapid polymerization of actin in the infracuticular region of the cell. The results of the present study imply that infracuticular actin in OHCs occurs in a particular, reproducible pattern along the organ of Corti of the guinea pig and its structure is neither the result of preparation procedures nor confined to contracted and degenerate isolated OHCs.

Actin is a ubiquitous protein in muscle and non-muscle cells. It exists in either the monomeric (G-actin) or polymerized (F-actin) form and the latter is seen by electron microscopy as microfilaments (5-7 $\mathrm{nm}$ in diameter). Phalloidin, with either a fluorescein or rhodamine label is a specific marker for F-actin and has been used to demonstrate its location in a variety of cell types, including cochlear hair cells (Drenkhahn et al., 1985; Zenner and Drenkhahn, 1986). Tetramethylrhodaminylphalloidin is a semi-synthetic phallotoxin (Faulstich et al., 1983) which has a lower affinity for F-actin than that with a fluorescein label but it is more resistant to photobleaching and is less toxic to cells (Faulstich et al., 1983).

Polymerized actin plays a role in numerous cellular functions in muscle and non-muscle cells. It may be involved in contraction, maintenance of cellular architecture, locomotion or motility, cell adhesion and orientation of specific organelles (Fulton, 1984). The extent of polymerization, and the binding and orientation of microfilaments is controlled by various proteins and may play an important role in modulating the degree of gela- 
tion of the cytoplasm (Pollard et al., 1982; De Rosier and Tilney, 1982; Stossel et al., 1982).

In hair cells, actin is considered to provide rigidity to stereocilia (Flock et al., 1977; Tilney et al., 1980), but it has been postulated that it could also be involved in the transduction process (Hudspeth. 1985). The presence of myosin (Drenkhahn et al., 1982) and tropomyosin (Drenkhahn et al., 1985) in the cuticular plate has led to speculation that the stiffness of stereocilia may be regulated by a contractile process. Support for this comes from evidence that the resistance of stereocilia of guinea pig cochlear hair cells to displacement can be modified in vitro by a solution that induces contraction in muscle (Orman and Flock, 1983).

There is evidence that in vitro the hair cell body shortens when given the appropriate stimulus (Brownell et al., 1985; Zenner et al., 1985: Flock et al. 1986; Kachar et al., 1986; Zenner, 1986b). The mechanism for this process is not known but it has been suggested that contraction may occur by an interaction of contractile proteins in the cell cytoplasm (Zenner et al., 1985; Flock et al., 1986; Zenner and Drenkhahn, 1986), or by an electrokinetic process involving the movement of intracellular water during electrical stimulation (Brownell and Kachar, 1985; Kachar et al., 1986). In favour of the first mechanism are the observations that contractions can be induced by an elevation of intracellular calcium (in the presence of ATP) and that they may be controlled by the phosphoinositide second messenger system (Schacht and Zenner, 1986). The infracuticular actin filaments may be part of a cytoplasmic network of contractile proteins involved in the motility of $\mathrm{OHC}$. The interaction of myosin and actin within the cuticular plate may pull the infracuticular and subplasmalemmal actin filaments towards the cuticular plate resulting in a shortening or change in tonicity of the cell body (Zenner and Drenkhahn, 1986). Infracuticular filaments could also interact directly with other cytoskeletal proteins in the same region but further study is required to determine whether other cytoskeletal proteins are associated with the infracuticular network. It is interesting that the infracuticular filaments are associated with endoplasmic reticulum and mitochondria, suggestive of an energy and cation dependent contractile process, as in muscle cells. In vivo contraction, or a change in stereocilia or cell body stiffness would have important consequences for the control of basilar membrane mechanical characteristics (Kim, 1984).

If the infracuticular actin filaments are associated directly with hair cell motility, then by inference from their distribution such a function would be confined to OHCs in more apical turns. This would conflict with the suggestion that it is the OHCs in the basal turn that may serve more as effector cells (Dallos, 1985b). However, changes in length or tonicity of basal OHCs could occur by a different mechanism, such as by the rapid polymerization of actin from a cytoplasmic pool of G-actin (Zenner, 1986b).

It cannot be ruled out that the infracuticular filaments have a purely structural function. OHC length increases from base to apex and from 1st to 3 rd row (Smith, 1968) in a similar pattern of distribution to the infracuticular network. In association with the ring around the cuticular plate. the infracuticular network could provide an internal skeleton to hold the longer more apical OHCs in a particular configuration. This could be important to maintain the mechanical characteristics of the cochlear partition in apical turns where the organ of Corti becomes particularly large.

In conclusion, infracuticular F-actin in OHC. of the organ of Corti is differentially distributed according to the radial and longitudinal location of the cell. This provides further evidence that the OHCs of the guinea pig organ of Corti are not a structurally homogencous population of cells but that there are substantial differences along the organ of Corti. Although it is presently not possible to relate this difference in distribution of $F$ actin to the postulated differences in function, these results are intriguing in that they suggest a structural basis for functional differences of $\mathrm{OHC} s$ along the organ of Corti. Further studies of the distribution of other cytoskeletal proteins may be useful in establishing the function of this infracuticular network.

\section{Acknowledgements}

The authors wish to thank Dr. A.L. Nuttall and Professor J.E. Hawkins, Jr.. for valuable advice 
during this study and constructive criticisms of the manuscript. The assistance of Mrs. J. Hardy in producing the manuscript and the technical assistance of Mrs. T.E. Billett are gratefully acknowledged. This research was supported by NIH Program Project Grant NS 05785, NIH grant 11731, and by a USPHS International Research Fellowship no. TWO 3473 to P.R.T.

\section{References}

Barak, L.S., Yocum, R.R. and Webb, W.W. (1981) In vivo staining of cytoskeletal actin by autointernalization of nontoxic concentrations of nitrobenzoxadiazole-phallacidin. J. Cell Biol. 89, 368-372.

Brownell, W.E. and Kachar, B. (1985) Outer hair cell motility: A possible electro-kinetic mechanism. In: Peripheral Auditory Mechanisms, pp. 369-376. Editors: J.B. Allen, J.L. Hall, A. Hubbard, S.T. Neely and A. Tubis. Springer-Verlag, New York.

Brownell, W.E., Bader, C.R., Bertrand. D. and De Ribaupierre, Y. (1985) Evoked mechanical response of isolated cochlear hair cells. Science 227, 194-196.

Dallos, P. (1985a) Response characteristics of mammalian cochlear hair cells. J. Neurosci. 5, 1591-1608.

Dallos, P. (1985b) The role of outer hair cells in cochlear function. In: Contemporary Sensory Neurobiology, pp. 207-230. Editors: M.J. Correia and A.A. Perachio. Alan Liss, New York.

Davis, H. (1983) An active process in cochlear mechanics. Hear Res. 9, 79-90.

DeRosier, D.J. and Tilney, L.G. (1982) How actin filaments pack into bundles. Cold Spring Harbor Symp. Quant. Biol. 66, 525-540.

Drenkhahn, D., Kellner, J., Mannherz, H.G., Groschel-Stewart, U., Kendrick-Jones, J. and Scholey, J. (1982) Absence of myosin-like immunoreactivity in stereocilia of cochlear hair cells. Nature 300, 531-532.

Drenkhahn, D., Schafer, T. and Prinz, M. (1985) Actin, myosin and associated proteins in the vertebrate auditory and vestibular organs: Immunocytochemical and biochemical studies. In: Auditory Biochemistry, pp. 317-335. Editor: D.G. Drescher. Thomas, Springfield, IL.

Faulstich, H., Trischmann, H. and Mayer, D. (1983) Preparation of tetramethylrhodaminyl-phalloidin and uptake of the toxin into short-term cultured hepatocytes by endocytosis. Exp. Cell Res. 144, 73-82.

Flock, $\AA$. (1985) Contractile and structural proteins in the auditory organ. In: Auditory Biochemistry, pp. 310-316. Editor: D.G. Drescher. Thomas, Springfield, IL.

Flock, $\AA$. and Cheung, H.C. (1977) Actin filaments in sensory hairs of inner ear receptor cells. J. Cell Biol. 75, 339-343.

Flock. A., Flock, B. and Murray, E. (1977) Studies on the sensory hairs of receptor cells in the inner ear. Acta OtoLaryngol. 83, 85-91.

Flock, A., Bretscher, A. and Weber, K. (1982) Immunohistochemical localization of several cytoskeletal proteins in inner ear sensory and supporting cells. Hear. Res. 6, 75-89.
Flock, A., Flock, B. and Ulfendahl, M. (1986) Mechanisms of movement in outer hair cells and a possible structural basis. Arch. Oto-Rhino-Laryngol. 243, 83-90.

Fulton, A.B. (1984) The Cytoskeleton. Cellular Architecture and Choreography. Chapman and Hall, New York.

Hayat, M.A. (1981) Fixation for Electron Microscopy. Academic Press, New York.

Hoshino, T. (1975) An electron microscopic study of the otolithic maculae of the lamprey (Entosphenus japonicus). Acta Oto-Laryngol. 80, 43-53.

Hudspeth, A.J. (1985) Models for mechanoelectrical transduction by hair cells. In: Contemporary Sensory Neurobiology, pp. 193-205. Editors: M.J. Carreia and A.A. Perachio. Alan Liss, New York.

Kachar, B., Brownell, W.C., Altschuler, R.A. and Fex, J. (1986) Electrokinetic shape changes of cochlear outer hair cells. Nature 322, 365-367.

Kim, D.O. (1984) Functional roles of the inner- and outer-hair-cell subsystems in the cochlea and brainstem. In: Hearing Science, pp. 241-262. Editor: C. Berlin. CollegeHill Press, San Diego.

Lim, D.J. and Melnick. W. (1971) Acoustic damage to the cochlea: A scanning and transmission electron microscopic observation. Arch. Otolaryngol. 94, 294-305.

Lowenstein, O. and Osborne, M.P. (1964) Ultrastructure of the sensory hair-cells in the labyrinth of the amniocoete larva of the lamprey, Lametra fluviatilis. Nature 204, 197-198.

Maupin-Szamier, P. and Pollard, T.D. (1978) Actin filament destruction by osmium tetroxide. J. Cell. Biol. 77, 837-852.

Neely, S.T. and Kin, D.O. (1983) An active cochlear model showing sharp tuning and high sensitivity. Hear. Res. 9. $123-130$.

Orman, S. and Flock, А. (1983) Active control of sensory hair mechanics implied by susceptibility to media that induce contraction in muscle. Hear. Res. 11, 261-266.

Pollard, T.D.. Aebi, U., Cooper, J.A., Fowler, W.E. and Tseng, P. (1982) Actin structure, polymerization and gelation. Cold Spring Harbor Symp. Quant. Biol. 66, 513-524.

Pollard, T.D., Selden, S.C. and Maupin, P. (1984) Interaction of actin filaments with microtubules. J. Cell. Biol. 99 . 33S-37S.

Pujol, R. and Lenoir, M. (1986) The four types of synapses in the organ of Corti. In: Neurobiology of Hearing: The Cochlea. pp. 161-172. Editors: R.A. Altschuler, D.W. Hoffman and R.P. Bobbin. Raven Press, New York.

Russell, I.J. and Cody, A.R. (1985) Transduction in cochlear hair cells. In: Peripheral Auditory Mechanisms, pp. 349-360. Editors: J.B. Allen, J.L. Hall, A. Hubbard, S.T. Neely and A. Tubis. Springer-Verlag, New York.

Saunders, J.C., Schneider, M.E. and Dear, S.P. (1985) The structure and function of actin in hair cells. $\mathbf{J}$. Acoust. Soc. Am. 78, 299-311.

Schacht, J. and Zenner, H.P. (1986) Phosphoinositides in isolated outer hair cells: Possible second messenger systems for motile responses. Abstr. Assoc. Res. Otolaryngol. 9. 2.

Slepecky, N. and Chamberlain, S.C. (1985) Immunoelectron microscopic and immunofluorescent localization of cytoskeletal and muscle-like contractile proteins in inner ear sensory hair cells. Hear Res. 20. 245-260. 
Smith, C.A. (1968) Ultrastructure of the organ of Corti. Adv. Sci. $24,419-433$.

Spoendlin, H. (1970) Auditory, vestibular, olfactory and gustatory organs. In: Ultrastructure of the Peripheral Nervous System and Sense Organs, pp. 173-338. Editor: A. Bischoff. Georg Thieme Verlag, Stuttgart.

Spoendlin, H. (1979) Sensory neural organization of the cochlea. J. Laryngol. Otol. 93, 853-877.

Stossel, T.P.. Hartwig, J.H., Yin, H.L., Zaner, K.S. and Stendahl. O.I. (1982) Actin gelation and the structure of cortical cytoplasm. Cold Spring Harbor Symp. Quant. Biol. 66. $569-578$.

Strelioff, D. and Flock, A. (1984) Stiffness of sensory-cell hair bundles in the isolated guinea pig cochlea. Hear. Res. 15. 19-28.

Tilney, L.G. and DeRosier, D.J. (1985) The organization of actin filaments in the stereocilia of the hair cells of the cochlea. In: Auditory Biochemistry, pp. 281-309. Editor: D.G. Drescher. Charles C. Thomas, Springfield. IL.

Tilney. L.G., DeRosier, D.J. and Mulroy, M.J. (1980) The organization of actin flaments in the stereocilia of cochlear hair cells. J. Cell Biol. 86, 244-259.
Wright, A. (1984) Dimensions of the cochlear stereocilia in man and the guinea pig. Hear. Res. 13.89 98.

Zajic. G. and Schacht. J. (1987) Comparison of isolated outer hair cells from five mammalian species. Hear. Res. 26, 249-256.

Zenner. H.P. (1980) Cytoskeletal and muscle-like elements in cochlear hair cells. Arch. Oto-Rhino-Laryngol. 230. 81-92.

Zenner, H.P. (1986a) Molecular structure of hair cells. In: Neurobiology of Hearing: The Cochlea, pp. 1-21. Editors: R.A. Altschuler, D.W. Hoffman and R.P. Bobbin. Raven Press, New York.

Zenner. H.P. (1986b) Motile response in outer hair cells. Hear. Res. 22.83 .90 .

Zenner, H.P, and Drenkhahn, D. (1986) Active motile events in guinea pig outer hair cells. Abstr. Assoc. Res. Otolaryngol. 9.1.

Zenner, H.P., Zimmermann, U. and Schmitt, U. (1985) Reversible contractions of isolated mammalian cochlear hair cells. Hear. Res. 18, 127-133. 